\title{
EVIDÊNCIAS DE VALIDADE PARA A ESCALA BAPTISTA DE DEPRESSÃO VERSÃO HOSPITAL-AMBULATÓRIO (EBADEP-HOSP-AMB)
}

\author{
Gabriela da Silva Cremasco \\ Universidade São Francisco
}

\author{
Makilim Nunes Baptista \\ Universidade São Francisco
}

\begin{abstract}
Resumo
O objetivo do estudo foi buscar evidências de validade baseadas na relação com variáveis externas para a Escala Baptista de Depressão Versão Hospital-Ambulatório (EBADEP-HOSP-AMB). Participaram 210 pacientes renais crônicos em hemodiálise, com idades entre 18 e 82 anos $(M=53,40 ; D P=14,40)$, sendo $112(53,3 \%)$ do sexo masculino. Foram aplicados um questionário sociodemográfico/saúde, juntamente com a EBADEP-HOSP-AMB, a Escala Hospitalar de Ansiedade e Depressão (HADS) e a Escala de Pensamentos Depressivos (EPD). As aplicações ocorreram de forma individual durante as sessões de hemodiálise. Foi verificada a relação entre os instrumentos além de possíveis diferenças de média em função das variáveis sociodemográficas. Os principais resultados indicaram correlações significativas de magnitudes moderadas a altas entre os instrumentos. Também foi observado que as mulheres e aqueles que relataram ter diagnóstico de depressão obtiveram escores mais elevados na maioria das escalas apresentando mais sintomatologia depressiva e pensamentos depressivos, bem como ansiedade.
\end{abstract}

Palavras-chave: sintomatologia depressiva; doença renal; evidências de validade.

\section{VALIDITY EVIDENCE FOR THE BAPTISTA DEVELOPMENTAL SCALE HOSPITAL-AMBULATORY VERSION (EBADEP-HOSP-AMB)}

\begin{abstract}
The purpose of the study was to seek validity evidence based on the relationship with external variables for the Hospital-Ambulatory Depression Baptist Scale (EBADEPHOSP-AMB). A total of 210 chronic kidney patients undergoing hemodialysis, aged $18-$ 82 years $(M=53.40, \quad S D=14.40), 112(53.3 \%)$ males participated. A sociodemographic/health questionnaire was applied, along with the EBADEP-HOSPAMB, the Hospital Anxiety and Depression Scale (HADS) and the Depressive Thoughts Scale (EPD). The applications occurred individually during the hemodialysis sessions. The relationship between the instruments was verified, besides possible differences of mean according to the sociodemographic variables. The main results indicated significant correlations of moderate to high magnitudes between the instruments. It was also observed that women and those who reported having a diagnosis of depression scored higher on most scales presenting more depressive symptomatology and depressive thoughts as well as anxiety.
\end{abstract}

Keywords: depressive symptomatology; kidney disease; evidence of validity. 


\title{
EVIDENCIAS DE VALIDEZ PARA LA ESCALA BAPTISTA DE DEPRESIÓN VERSIÓN HOSPITAL-AMBULATORIO (EBADEP-HOSP-AMB)
}

\begin{abstract}
Resumen
El objetivo del estudio fue buscar evidencias de validez basadas en la relación con variables externas para la Escala Baptista de Depresión Versión Hospital-Ambulatorio (EBADEP-HOSP-AMB. Participaron un total de 210 pacientes renales crónicos en hemodiálisis, con edades entre 18 y 82 años $(M=53,40 ; D P=14,40)$, siendo 112 $(53,3 \%)$ del sexo masculino. Se aplicó un cuestionario sociodemográfico / salud, junto con EBADEP-HOSP-AMB, la Escala Hospitalaria de Ansiedad y Depresión (HADS) y la Escala de Pensamientos Depresivos (EPD). Los cuestionarios fueron aplicados de forma individual durante las sesiones de hemodiálisis. Se verificó la relación entre los instrumentos además de posibles diferencias de promedio en función de las variables sociodemográficas. Los principales resultados indicaron correlaciones significativas de magnitudes moderadas a altas entre los instrumentos. También se observó que las mujeres y aquellos que reportaron tener diagnóstico de depresión obtuvieron puntuaciones más altas en la mayoría de las escalas presentando más sintomatología depresiva y pensamientos depresivos, así como ansiedad.
\end{abstract}

Palabras clave: sintomatología depresiva; enfermedad renal; evidencias de validez.

\section{INTRODUÇÃO}

Estimativas da World Health Organization (WHO, 2017) indicam que atualmente mais de 300 milhões de pessoas no mundo têm depressão, com impacto negativo sobre a saúde. A depressão é considerada a principal causa de mortes por suicídio, que chegam a, aproximadamente, 800 mil por ano. É caracterizada principalmente pela presença do humor deprimido e perda de interesse ou prazer, além de sentimento de culpa, baixa autoestima e baixa concentração, e tende a prejudicar de forma significativa o funcionamento diário das pessoas. As taxas de prevalência variam de acordo com a idade, com maior prevalência na idade adulta avançada. Acomete 7,5\% das mulheres com idades entre 55 a 74 anos e acima de 5,5\% dos homens nessa faixa etária, indicando que o transtorno é mais frequente em mulheres (WHO, 2017).

De acordo com o modelo cognitivo, os sintomas da depressão surgem a partir da interpretação que o sujeito faz de si, do mundo e do futuro (tríade cognitiva) conforme relatado por Beck, Rush, Shaw, e Emery (1997). Portanto, verificar a forma como as pessoas avaliam suas experiências auxilia na identificação da sintomatologia depressiva, tendo em vista que, quanto maior a sua gravidade, maior a frequência das interpretações negativas feitas pelo sujeito (Lynch, Moore, Moss-Moris, \& Kendrick, 2011).

Assim como a depressão, os transtornos de ansiedade também afetam pessoas no mundo todo e as estimativas indicam um total de 264 milhões de acometidos, sendo mais comum nas mulheres $(4,6 \%)$ do que nos homens $(2,6 \%)$. As taxas globais de depressão e ansiedade são de 4,4\% e 3,6\%, respectivamente. Além disso, muitas pessoas vivenciam esses transtornos de forma simultânea e ambas podem apresentar sintomatologia que varia de leve a grave (WHO, 2017). 
A ansiedade pode ser compreendida como um sentimento desagradável que proporciona reações de medo, apreensão, tensão e desconforto. Trata-se de uma condição relacionada ao futuro, isto é, a expectativa de um acontecimento negativo gera essas reações como uma resposta ao perigo iminente, real ou percebido. Quando deixa de ser um componente adaptativo e passa a ser desproporcional em relação à realidade, a ansiedade torna-se patológica, gerando consequências como diminuição da qualidade de vida, problemas emocionais e de desempenho nas atividades (Castillo, Recondo, Asbahr, \& Manfro, 2000; Pinto, Martins, Pinheiro, \& Oliveira, 2015).

Watson (2009) denotou que as pesquisas sobre a relação da ansiedade com a depressão têm recebido atenção ao longo dos anos, sendo obtidas fortes associações em amostras de crianças, universitários e populações clínicas. A diferenciação entre esses construtos é descrita como complexa em decorrência da grande associação entre sintomas da ansiedade e depressão. Além disso, há a sobreposição de sintomas específicos de cada uma, ocasionando problemas nos instrumentos de avaliação, que apresentam correlações fortes, mas discriminação moderada (Clark \& Watson, 1991; Watson et al., 1995).

Para elucidar a relação entre sintomas de ansiedade e de depressão, Clark e Watson (1991) propuseram a chamada teoria tripartite. Esta teoria parte do pressuposto que, para uma descrição minuciosa do domínio afetivo, é necessário verificar tanto os elementos únicos de cada uma das síndromes como os seus elementos em comum. Desse modo, pessoas com transtornos depressivos poderiam apresentar sintomatologia de ansiedade ou vice-versa. De acordo com esse modelo, a ansiedade e a depressão compartilham um elemento comum, o afeto negativo, que corresponde à sobreposição dos sintomas. Afeto negativo refere-se a um conjunto de estados como raiva, culpa, medo, tristeza, desprezo, preocupação, angústia, dentre outros, sendo que os estados de calma e relaxamento representariam uma ausência desse afeto. Indivíduos com sintomatologia depressiva tendem a apresentar poucos afetos positivos e muitos afetos negativos, ao passo que, pessoas com sintomas de ansiedade, tendem a manifestar altos níveis de excitação fisiológica e altos níveis de afetos negativos (Clark \& Watson, 1991).

Assim, a diferenciação entre a depressão e ansiedade ocorre em razão de fatores específicos de cada um desses transtornos, como a anedonia, que se refere a um aspecto específico da depressão, que corresponde à perda do prazer. A excitação fisiológica ou hiperestimulação, diz respeito ao quesito característico da ansiedade, sendo representada por tensões somáticas, dispneia, tonturas, vertigens e boca seca (Anderson \& Hope, 2008; Clark \& Watson, 1991). Embora os estudos apontem a associação das doenças crônicas em geral com transtornos mentais, a Doença Renal Crônica (DRC), especificamente, tem maior relação com a presença de transtornos como depressão e ansiedade, apresentando taxas que 
variam de $20 \%$ e $30 \%$ para depressão e de $30 \%$ e $45 \%$ para ansiedade em pacientes em hemodiálise (Feroze et al., 2012).

Theofilou (2011) realizou um estudo com objetivo de verificar a relação da depressão e ansiedade com características sociodemográficas em uma amostra de 144 pacientes com doença renal crônica. Os participantes responderam ao General Health Questionnaire (GHQ-28), a Center for Epidemiologic Studies Depression Scale (CES-D) e a State-Trait Anxiety Inventory (STAI I/STAI II). Os principais resultados indicaram que as mulheres apresentaram mais sintomatologia depressiva na subescala de depressão do GHQ e também mais sintomas de ansiedade. Além disso, os pacientes com menor escolaridade, com idade superior a 45 anos, divorciados e viúvos, tiveram escores mais altos na escala de depressão.

Dias, Shiozawa, Miorin, e Cordeiro (2015) avaliaram a prevalência de sintomas de depressão e ansiedade em pacientes em hemodiálise por meio da Escala Hospitalar de Ansiedade e Depressão (HADS). Participaram 81 pacientes, sendo $41(50,62 \%)$ do sexo feminino. Quanto à etiologia da doença, a maior parte $(60,4 \%)$ não tinha especificação, $18,5 \%$ em decorrência da hipertensão arterial, $11,1 \%$ devido à glomerulonefrite crônica e 9,8\% por conta da diabetes. A principal comorbidade identificada foi a hipertensão arterial (53,0\%), juntamente com a associação de hipertensão e diabetes $(18,5 \%)$ e diabetes melitus $(3,0 \%)$. Os resultados demonstraram que $19(23,4 \%)$ pacientes do estudo apresentaram sintomas de depressão e $17(20,9 \%)$ de ansiedade.

Stasiak, Bazan, Kuss, Schuinski, e Baroni (2014) também avaliaram a prevalência de depressão e ansiedade em pacientes renais. Dos 155 participantes, 128 estavam em tratamento de hemodiálise, sendo $70(54,7 \%)$ do sexo masculino, com idade média de 54,95 anos e 27 em diálise peritoneal. Foram aplicados o Inventário de Depressão de Beck (BDI), o Inventário de Ansiedade de Beck (BAI) e a Escala Hospitalar de Ansiedade e Depressão (HADS). Entre os pacientes em hemodiálise, foram identificados sintomas de depressão em 22,6\% segundo o BDI e em 9,3\% pela HADS-depressão e de ansiedade em $25,7 \%$ pelo BAI e em $11,7 \%$ a partir da HADS-ansiedade. Não foram observadas diferenças significativas em relação ao sexo para ambos os construtos. A prevalência de depressão foi maior quando avaliada pelo BDI em relação a HADS-depressão, o que pode ter ocorrido devido ao fato de o BDI verificar também os sintomas vegetativos associados à doença renal, o que pode enviesar os dados pelo tipo da amostra avaliada.

É importante ponderar que pacientes com alguma doença têm maior probabilidade de apresentarem sintomas somáticos comuns a depressão em decorrência da sua própria condição clínica (Baptista, 2012). Assim, é necessário que os instrumentos que tenham como objetivo avaliar a sintomatologia depressiva no ambiente hospitalar/ambulatorial sejam construídos pensando nas especificidades desse contexto, não existindo ainda uma ferramenta normatizada 
para o contexto brasileiro. A Escala Baptista de Depressão (Versão HospitalAmbulatório) - EBADEP-HOSP-AMB (Baptista, 2013) foi desenvolvida com base em descritores da depressão. No entanto, não apresenta em sua composição sintomas vegetativos/somáticos como diminuição ou aumento do apetite, insônia ou hipersonia, agitação ou retardo psicomotor e fadiga ou perda de energia. Alguns estudos foram realizados com o instrumento, sendo obtidas evidências de validade baseada na relação com variáveis externas para a escala (Marques, 2016; Messias, 2014).

Nesse tipo de evidência, busca-se a relação entre os escores do instrumento e outras variáveis medindo a mesma característica, características relacionadas ou características diferentes, sendo que outras variáveis podem ser sexo, idade, critérios diagnósticos, dentre outras (American Educational Research Association, American Psychological Association, National Council on Measurement in Education [AERA, APA, NCME], 2014). Conforme os critérios estabelecidos por Prieto e Muñiz (2000), considera-se adequadas correlações com magnitudes superiores a 0,40 e excelentes aquelas com magnitudes a partir de 0,60 em se tratando de instrumentos similares. No entanto, não são apresentados valores esperados para construtos relacionados.

A partir do exposto, os objetivos desta pesquisa foram: buscar evidências de validade baseadas na relação com variáveis externas para EBADEP-HOSPAMB, juntamente com a Escala Hospitalar de Ansiedade e Depressão (HADS) e a Escala de Pensamentos Depressivos (EPD); verificar a prevalência de sintomatologia depressiva, pensamentos depressivos e sintomas de ansiedade da amostra, além de possíveis diferenças de média em relação às variáveis sociodemográficas.

\section{MÉTODO}

\section{Participantes}

Foram participantes da pesquisa 210 pacientes com Doença Renal Crônica (DRC) em tratamento de hemodiálise, escolhidos por conveniência, de três instituições de saúde públicas e privadas de municípios do interior do estado de São Paulo. A idade dos pacientes variou entre 18 e 82 anos $(M=53,40$; $\mathrm{DP}=14,40)$ e $11(5,2 \%)$ relataram diagnóstico atual de depressão. O tempo de tratamento em hemodiálise variou de 1 mês a 28 anos. Foram levantados dados referentes a outras questões sociodemográficas e de saúde, tais como escolaridade, sexo, estado civil, trabalho, causa da doença e presença de comorbidades (Tabela 1 ). 
Tabela 1.

Caracterização sociodemográfica da amostra de pacientes renais crônicos em hemodiálise

\begin{tabular}{|c|c|c|}
\hline Variável & Categoria & N (\%) \\
\hline \multirow[t]{2}{*}{ Sexo } & Masculino & $112(53,3)$ \\
\hline & Feminino & $98(46,7)$ \\
\hline \multirow[t]{7}{*}{ Escolaridade } & Analfabetos & $8(3,8)$ \\
\hline & Fundamental Incompleto & $50(23,8)$ \\
\hline & Fundamental Completo & $69(32,9)$ \\
\hline & Médio Incompleto & $11(5,2)$ \\
\hline & Médio Completo & $51(24,3)$ \\
\hline & Superior Incompleto & $3(1,4)$ \\
\hline & Superior Completo & $18(8,6)$ \\
\hline \multirow[t]{5}{*}{ Estado Civil } & Solteiro & $49(23,3)$ \\
\hline & Casado & $97(46,2)$ \\
\hline & Divorciado & $31(14,8)$ \\
\hline & Viúvo & $21(10,0)$ \\
\hline & Relação estável & $12(5,7)$ \\
\hline \multirow[t]{8}{*}{ Causa da Doença } & Sem especificação & $34(16,2)$ \\
\hline & Pressão alta & $82(39,0)$ \\
\hline & Diabetes & $20(9,5)$ \\
\hline & Pressão alta e diabetes & $27(12,9)$ \\
\hline & Glomerulonefrite & $11(5,2)$ \\
\hline & Rim policístico & $8(3,8)$ \\
\hline & Lúpus & $3(1,4)$ \\
\hline & Outros & $25(11,9)$ \\
\hline \multirow[t]{9}{*}{ Outras doenças } & Não & $78(37,1)$ \\
\hline & Sim & $132(62,9)$ \\
\hline & Se sim, qual & \\
\hline & Pressão alta & $69(32,9)$ \\
\hline & Diabetes & $16(7,6)$ \\
\hline & Pressão alta e diabetes & $36(17,1)$ \\
\hline & HIV & $5(2,4)$ \\
\hline & Lúpus & $4(1,9)$ \\
\hline & Outros & $2(1,0)$ \\
\hline
\end{tabular}

\section{Instrumentos}

Questionário Sociodemográfico/Saúde: composto por questões que investigam idade, sexo, estado civil, escolaridade, causa da doença, tempo de tratamento, se tem alguma outra doença e diagnóstico atual de depressão.

Escala Baptista de Depressão (Versão Hospital-Ambulatório) (EBADEPHOSP-AMB; Baptista, 2013): trata-se de um instrumento de rastreamento da sintomatologia depressiva, direcionada a amostras ambulatoriais/hospitalares. 
Foi construído a partir de indicadores sintomáticos de depressão provenientes de teorias da depressão, como a teoria Cognitiva (Beck et al., 1997) e a analíticocomportamental (Ferster, Culbertson, \& Boren, 1977), além dos manuais internacionais de diagnóstico da Associação Americana de Psiquiatria, DSM-IV-TR (APA, 2002) e da Organização Mundial da Saúde, CID-10 (OMS, 1993).

O instrumento é derivado dos descritores da Escala Baptista de Depressão (Versão Adulto) - EBADEP-A (Baptista, 2012) e exclui os descritores vegetativos, tais como fadiga e sono, por exemplo. A análise preliminar da estrutura interna por meio da análise fatorial exploratória indicou uma solução de dois fatores, com variância total de 56\%. A escala ficou composta por 20 itens, em escala do tipo Likert de três pontos, de zero a dois, sendo que o Fator 1, denominado de "Aspectos negativos" (14 itens), apresentou confiabilidade de 0,95 e o Fator 2, chamado de "Aspectos positivos" ( 6 itens), indicou confiabilidade de 0,89. Foram verificados bons índices de ajuste para os fatores pela Teoria de Resposta ao Item (TRI) (Cremasco \& Baptista, 2017). Exemplos de itens da escala: "Sinto-me cada vez mais sozinha (o)"; "Gosto da minha aparência"; "Tenho chorado muito"; "Gosto de mim".

Escala de Pensamentos Depressivos (EPD; Carneiro \& Baptista, 2016): o instrumento tem como objetivo avaliar o quanto o indivíduo apresenta distorções a respeito de si e do futuro, e o quão funcional é sua percepção a respeito das pessoas com quem convive. Contém 26 itens, em escala do tipo Likert de três pontos, de um a três, divididos em dois fatores: Fator 1 (Baixa autoestima/desesperança) e Fator 2 (Funcionalidade nas relações), que deve ser pontuado de forma inversa. A escala foi construída com base na tríade cognitiva de Beck et al. (1997). Foram realizados estudos de evidências de validade e precisão com amostras do estado de São Paulo e Minas Gerais, e fizeram parte das pesquisas estudantes universitários, trabalhadores administrativos, profissionais de saúde, bem como pacientes com diagnóstico de depressão confirmado pela Structured Clinical Interview for DSM-IV (SCID) (First, Spitzer, Gibbon, \& Williams, 1996). Em relação aos índices de confiabilidade, o Fator 1 apresentou alfa de Cronbach de 0,93 e o Fator 2 alfa com valor de 0,89.

Escala Hospitalar de Ansiedade e Depressão (HADS; Zigmond \& Snaith, 1983): o instrumento foi adaptado para o Brasil por Botega, Bio, Zomignani, Garcia Júnior, e Pereira (1995) e tem o intuito de avaliar graus leves de sintomas de ansiedade e/ou depressão em ambientes não psiquiátricos. A escala é composta por 14 itens, com respostas do tipo Likert de quatro pontos, de zero a três, sendo dividida em duas subescalas: metade dos itens avaliam ansiedade (HADS-A) e os demais avaliam depressão (HADS-D), com pontuação mínima de zero e máxima de 21 pontos para cada subescala. Foi adotado o ponto de corte $\geq 9$ para as duas subescalas, como indicado pelos autores da escala. Os índices de confiabilidade das subescalas de ansiedade e depressão apresentaram alfa de 0,84 e 0,83, respectivamente. 


\section{Procedimentos}

Após a autorização das instituições e aprovação do projeto pelo Comitê de Ética em Pesquisa da Universidade São Francisco (CAAE: 59264816.7.0000.5514), os participantes responderam aos instrumentos mediante assinatura do Termo de Consentimento Livre e Esclarecido (TCLE), impresso em duas vias, no qual constavam todas as informações pertinentes ao estudo em questão. Os instrumentos foram aplicados de forma individual durante as sessões de hemodiálise, sendo que os itens foram lidos para os pacientes devido a condição física deles no momento do tratamento. Foram aplicados o Questionário Sociodemográfico/Saúde, seguido da EBADEP-HOSP-AMB, a EPD e a HADS, em uma duração aproximada de 30 minutos.

Os dados foram analisados por meio do programa Statistic Package for Social Sciences - SPSS (versão 21). O teste de correlação de Pearson foi utilizado para verificar a relação entre os construtos. Para avaliar possíveis diferenças de média foi utilizado o teste $t$ de Student (dois grupos) e o tamanho do efeito verificado pelo $d$ de Cohen: efeito pequeno se $(0,20 \leq d<0,50)$, médio se $(0,50 \leq$ $d<0,80)$ e grande se $(d \geq 0,80)$, sendo adotado nível de significância de 0,05.

\section{RESULTADOS}

A primeira análise apresenta a classificação dos pacientes conforme a pontuação bruta na EPD. O nível de pensamentos depressivos é dividido em baixo, médio-baixo, médio-alto e alto, conforme as normas da escala. Os resultados referentes a essa análise podem ser observados na Tabela 2.

Tabela 2.

Classificação dos pacientes renais crônicos em hemodiálise segundo as normas da EPD

\begin{tabular}{ccc}
\hline Classificação & $\begin{array}{c}\text { Baixa } \\
\text { autoestima/desesperança }\end{array}$ & $\begin{array}{c}\text { Funcionalidade nas } \\
\text { relações }\end{array}$ \\
\hline Baixo & $66(31,43 \%)$ & $129(61,43 \%)$ \\
Médio Baixo & $33(15,71 \%)$ & $39(18,57 \%)$ \\
Médio Alto & $39(18,57 \%)$ & $24(11,43 \%)$ \\
Alto & $72(34,29 \%)$ & $18(8,57 \%)$ \\
\hline
\end{tabular}

Os dados da Tabela 2 demonstram que no fator 1 "Baixa autoestima/desesperança" um total de 111 (52,86\%) pacientes ficaram alocados nas categorias médio-alto/alto. Já no fator 2 "Funcionalidade nas relações", 42 $(20,0 \%)$ pessoas se localizaram nas categorias médio-alto/alto. Isso indica que aproximadamente metade da amostra apresentou alta frequência de pensamentos depressivos em relação a si próprio, bem como uma visão negativa em relação ao futuro. Foi observada uma frequência menor de pacientes nas categorias citadas em relação ao segundo fator, denotando um menor número de 
pacientes com pensamentos negativos em relação ao mundo e pessoas de sua convivência. Comparadas ao primeiro fator, no entanto, esses dados são relevantes, visto que $20 \%$ da amostra pontuou alto no segundo fator.

Em seguida, foi analisada a prevalência de sintomatologia depressiva e ansiosa pela HADS, conforme o ponto de corte $\geq 9$. Os resultados indicaram que $62(29,5 \%)$ pacientes apresentaram sintomatologia depressiva e que o mesmo número de pacientes apresentou sintomas de ansiedade (29,5\%). Verificou-se a relação entre os construtos, sendo encontradas correlações positivas entre as escalas com magnitudes moderadas a fortes conforme a classificação proposta por Dancey e Reidy (2006). Os valores das correlações são apresentados na Tabela 3.

Tabela 3.

Correlações entre a EBADEP-HOSP-AMB, EPD e HADS

\begin{tabular}{|c|c|c|c|c|}
\hline Escala & $\begin{array}{c}\text { EPD-Baixa } \\
\text { autoestima/ } \\
\text { desesperança }\end{array}$ & $\begin{array}{c}\text { EPD } \\
\text { Funcionalidad } \\
\text { e nas relações }\end{array}$ & HADS-D & HADS-A \\
\hline
\end{tabular}
EBADEP-HOSP-AMB
$0,81 * *$
$0,56 * *$
$0,79 * *$
$0,70 * *$

Fator 1

$\begin{array}{lllll}\text { EBADEP-HOSP-AMB } & 0,62 * * & 0,54 * * & 0,62 * * & 0,46 * *\end{array}$

Fator 2

$\begin{array}{lllll}\text { EBADEP-HOSP-AMB } & 0,83 * * & 0,60 * * & 0,81 * * & 0,70 * *\end{array}$

Total

Nota. $* * \mathrm{p}<0,01$. Fatores da EBADEP-HOSP-AMB: F1 Aspectos Negativos; F2 Aspectos Positivos

Conforme apresentado na Tabela 3, o fator 1 da EBADEP-HOSP-AMB apresentou correlações fortes com a EPD-Baixa autoestima/desesperança, HADSD e HADS-A e moderada com a EPD-Funcionalidade nas relações, demonstrando que o aumento de sintomas da depressão se relacionou de forma positiva com os pensamentos depressivos e ansiedade. Já no fator 2 da EBADEP-HOSP-AMB foram obtidas correlações moderadas com a EPD-Baixa autoestima/desesperança e EPD-Funcionalidade nas relações e com a HADS-A e HADS-D. Por fim, as correlações da EBADEP-HOSP-AMB-Total com os demais instrumentos foram fortes, com exceção a EPD-Funcionalidade nas relações, que foi moderada. $O$ teste $\mathrm{t}$ de Student foi utilizado para identificar possíveis diferenças de média entre os pacientes na EBADEP-HOSP-AMB, EPD e HADS em função do sexo, sendo encontradas diferenças significativas. Os resultados obtidos podem ser observados na Tabela 4. 
Tabela 4.

Análise de diferença de média dos escores da EBADEP-HOSP-AMB, HADS e EPD de pacientes renais crônicos em hemodiálise em função do sexo

\begin{tabular}{cccccccc}
\hline & Sexo & Média & $\begin{array}{c}\text { Desvio } \\
\text { Padrão }\end{array}$ & $t$ & gl & $p$ & $\mathrm{~d}$ \\
\hline EBADEP-HOSP-AMB-Total & $\mathrm{F}$ & 15,07 & 11,25 & 2,900 & 193,232 & $0,004 *$ & 0,40 \\
& $\mathrm{M}$ & 10,83 & 9,74 & & & & \\
EBADEP-HOSP-AMB- & $\mathrm{F}$ & 12,72 & 9,35 & 2,949 & 190,553 & $0,004 *$ & 0,41 \\
Aspectos Negativos & $\mathrm{M}$ & 9,18 & 7,87 & & & & \\
EBADEP-HOSP-AMB- & $\mathrm{F}$ & 2,35 & 2,99 & 1,799 & 208 & 0,074 & 0,25 \\
Aspectos Positivos & $\mathrm{M}$ & 1,65 & 2,61 & & & & \\
HADS-Ansiedade & $\mathrm{F}$ & 7,70 & 5,54 & 3,641 & 208 & $0,001 *$ & 0,50 \\
& $\mathrm{M}$ & 5,11 & 4,79 & & & & \\
HADS- Depressão & $\mathrm{F}$ & 7,01 & 5,03 & 2,765 & 208 & $0,006 *$ & 0,38 \\
& $\mathrm{M}$ & 5,17 & 4,61 & & & & \\
EPD-Baixa & $\mathrm{F}$ & 24,09 & 9,42 & 2,375 & 185,385 & $0,019 *$ & 0,33 \\
autoestima/desesperança & $\mathrm{M}$ & 21,27 & 7,54 & & & & \\
EPD-Funcionalidade nas & $\mathrm{F}$ & 15,65 & 4,88 & 0,952 & 208 & 0,342 & 0,13 \\
relações & $\mathrm{M}$ & 15,00 & 5,02 & & & & \\
\hline Nota. F: Feminino (n=98); M: Masculino (n=112);*p<0,05 & & & &
\end{tabular}

Como mostra a Tabela 4, as mulheres tiveram pontuações superiores em relação aos homens em todos os instrumentos, indicando que elas apresentaram mais sintomas depressivos, pensamentos de depressão e ansiedade comparadas ao sexo masculino. O tamanho do efeito variou de pequeno a médio (Cohen, 1988). As diferenças observadas foram significativas, com exceção ao fator Aspectos Positivos da EBADEP-HOSP-AMB e ao fator Funcionalidade nas relações da EPD, que apresentaram $p$ maior que 0,05. Em seguida, comparou-se também as diferenças de média nas escalas em função de ter relatado diagnóstico de depressão atual. Os resultados estão apresentados na Tabela 5.

Tabela 5.

Análise de diferença de média dos escores da EBADEP-HOSP-AMB, HADS e EPD de pacientes renais crônicos em hemodiálise em função do diagnóstico de depressão

\begin{tabular}{ccccccccc}
\hline & $\begin{array}{c}\text { Diagnóstic } \\
\text { o atual de } \\
\text { depressão }\end{array}$ & $n$ & $M$ & D.P. & $t$ & $g l$ & $p$ & $d$ \\
\hline EBADEP-HOSP- & Sim & 11 & 24,3 & 13,16 & 3,81 & 208 & 0,001 & 1,0 \\
AMB-Total & Não & 199 & 6 & 10,17 & 1 & & $*$ & 4 \\
& & & 12,1 & & & & & \\
EBADEP-HOSP- & Sim & 11 & 18,9 & 9,51 & 3,21 & 208 & 0,002 & 0,9
\end{tabular}




\begin{tabular}{ccccccccc}
$\begin{array}{c}\text { AMB- Aspectos } \\
\text { Negativos }\end{array}$ & Não & 199 & 1 & 8,52 & 2 & & $*$ & 4 \\
& & & 10,3 & & & & & \\
EBADEP-HOSP- & Sim & 11 & 5,45 & 4,46 & 4,40 & 10,37 & 0,021 & 1,0 \\
$\begin{array}{c}\text { AMB- Aspectos } \\
\text { Positivos }\end{array}$ & Não & 199 & 1,78 & 2,57 & 1 & 1 & $*$ & 1 \\
HADS- & Sim & 11 & 12,7 & 6,62 & 4,28 & 208 & 0,001 & 1,1 \\
Ansiedade & Não & 199 & 3 & 5,01 & 3 & & $*$ & 5 \\
& & & 5,96 & & & & & \\
HADS- & Sim & 11 & 11,1 & 6,05 & 3,69 & 208 & 0,001 & 1,0 \\
Depressão & Não & 199 & 8 & 4,67 & 9 & & $*$ & 1 \\
EPD-Baixa & Sim & 11 & 31,8 & 9,83 & 3,78 & 208 & 0,001 & 1,0 \\
autoestima/ & Não & 199 & 2 & 8,22 & 6 & & $*$ & 8 \\
desesperança & & & 22,0 & & & & & \\
EPD- & Sim & 11 & 16,5 & 5,61 & 0,85 & 208 & 0,395 & 0,2 \\
Funcionalidade & Não & 199 & 5 & 4,92 & 2 & & & 5 \\
nas relações & & & 15,2 & & & & & \\
\hline
\end{tabular}

Nota. $* p<0,05$

Os resultados referentes a Tabela 5 demonstram que as pessoas que relataram estar diagnosticadas com depressão tiveram pontuações superiores em relação aos que não indicaram ter o diagnóstico, no entanto, é necessário realizar a análise com uma amostra mais pareada, tendo em vista o $\mathrm{n}$ baixo de pessoas que relataram diagnóstico de depressão. Foi verificado um tamanho de efeito grande em todos os instrumentos, com exceção ao fator Funcionalidade nas relações da EPD, que apresentou $p$ maior que 0,05 e um tamanho de efeito pequeno. Em relação a escolaridade, estado civil, idade e presença de outras doenças não foram obtidas diferenças significativas em nenhum dos construtos mensurados.

\section{DISCUSSÃO}

Tendo em vista os objetivos do presente estudo, foi possível encontrar evidências de validade baseada na relação com variáveis externas para a Escala Baptista de Depressão (Versão Hospital-Ambulatório) - EBADEP-HOSP-AMB. Os dados obtidos a partir do questionário sociodemográfico e de saúde indicaram que em relação a etiologia da doença renal, uma das principais causas foi a pressão alta. A maior parte da amostra relatou a presença de comorbidades, sendo as mais presentes a pressão alta, seguida da pressão alta e a diabetes juntas, demonstrando que esses dados foram semelhantes aos encontrados no estudo de Dias et al. (2015). Bastos, Bregman, e Kirsztajn (2010) relataram que 
dentre as pessoas com maior risco para DRC, encontram-se os hipertensos e os diabéticos.

De forma geral, uma boa parte da amostra apresentou pensamentos negativistas, com baixa autoestima e desesperança, além de, mesmo que em uma porcentagem menor, uma visão negativa frente ao mundo e nos relacionamentos interpessoais, com base na EPD. Tendo em vista a complexidade em avaliar a depressão em pessoas com questões médicas associadas, o diagnóstico deve ser pautado principalmente nos aspectos psicológicos da depressão, tais como anedonia, culpa, baixa autoestima e desesperança, por exemplo (Bautovich, Katz, Smith, Loo, \& Harvey, 2014). Além disso, avaliar a visão que as pessoas têm de si, do mundo e do futuro pode contribuir para identificar possíveis sintomas depressivos visto que, quanto mais grave a depressão, maior a tendência do indivíduo a apresentar uma avaliação negativa (Beck et al., 1997).

Aproximadamente um terço dos pacientes apresentou sintomatologia de depressão e ansiedade conforme os critérios da HADS. Esse resultado corrobora o relatado por Feroze et al. (2012) que indicaram que, em se tratando de pacientes renais crônicos, a prevalência de sintomatologia depressiva tende a variar entre $20 \%$ e $30 \%$. Apesar de frequente, a ansiedade em pacientes renais é pouco estudada. A cronicidade da doença e a hemodiálise tendem a ocasionar estresse constante. Além disso, esses pacientes se percebem intimidados, com sentimentos ambíguos entre o medo da vida e da morte (Dias et al., 2015).

As análises de correlação entre os fatores da EBADEP-HOSP-AMB e sua pontuação total, a EPD-Baixa autoestima/desesperança, EPD-Funcionalidade nas relações, HADS-A e HADS-D, demonstraram que todas as correlações foram significativas com magnitudes que variaram de moderadas a fortes, indicando que o aumento da sintomatologia depressiva se relacionou positivamente com os pensamentos depressivos e sintomas de ansiedade da amostra (Dancey \& Reidy, 2006). Conforme os critérios relacionados aos valores de magnitude das correlações estabelecidos por Prieto e Muñiz (2000), no presente estudo, a maior parte das correlações foram consideradas excelentes.

As variáveis sociodemográficas foram analisadas e comparadas com os resultados encontrados na literatura. Como exemplo, foi observado no presente estudo que as mulheres apresentaram maior média no fator Baixa autoestima/desesperança da EPD quando comparadas aos homens. Esse fator é caracterizado pelos pensamentos de que tudo dará errado, de sentir-se um perdedor, de incapacidade na resolução de problemas e de ser ajudado por alguém, indicando que as mulheres apresentaram mais sentimentos de incapacidade e inadequação do que os homens. As mulheres também pontuaram mais no fator Aspectos Negativos e na EBADEP-HOSP-AMB em sua pontuação total, além da subescala de ansiedade e depressão da HADS. Isso indica que o sexo feminino apresentou maior sintomatologia depressiva em ambos os 
instrumentos, além de mais sintomas de ansiedade. Esse resultado vai ao encontro da literatura, que indica que as mulheres, de forma geral, tendem a ter mais depressão e ansiedade do que os homens (AERA, APA, NCME], 2014; WHO, 2017). As diferenças do presente estudo em relação a ansiedade e depressão entre homens e mulheres foram semelhantes as encontradas por Theofilou (2011) que também obteve resultados significativos em uma amostra de doentes renais. No entanto, os resultados da pesquisa de Stasiak et al. (2014) vão de encontro aos do presente estudo, não sendo encontradas diferenças significativas entre os instrumentos no que se refere a essa variável.

Apesar de constituírem um grupo pequeno, os pacientes que relataram ter diagnóstico atual de depressão apresentaram mais sintomatologia depressiva e pensamentos depressivos, além de mais sintomas de ansiedade em relação aos que não indicaram terem o diagnóstico. Lynch et al. (2011) ponderaram que, quanto mais grave os sintomas de depressão, mais negativas são as avaliações que a pessoa faz. Além disso, como elucidado por Clark e Watson (1991), a depressão e a ansiedade compartilham o chamado afeto negativo, que inclui culpa, medo, tristeza, desprezo, angústia, dentre outros, sendo que pessoas com sintomatologia de depressão também podem apresentar sintomas de ansiedade ou vice-versa, o que torna difícil a distinção entre esses transtornos. Uma das possibilidades então, seria avaliar a anedonia, por exemplo, que corresponde a um sintoma característico da depressão (Anderson \& Hope, 2008; Clark \& Watson, 1991).

\section{CONSIDERAÇÕES FINAIS}

A doença renal crônica tem sido indicada na literatura como uma das patologias mais associadas a depressão. No entanto, apesar da alta prevalência, muitos pacientes são diagnosticados incorretamente, sendo muitas vezes utilizadas medidas inadequadas para o contexto, enviesando os resultados obtidos. Assim, ter instrumentos que avaliem a sintomatologia depressiva é importante, sendo a EBADEP-HOSP-AMB um instrumento promissor. A partir da avaliação correta dos sintomas, é possível desenvolver estratégias interventivas adequadas para o contexto em questão.

É importante considerar as limitações da presente pesquisa, quais sejam, a ausência de um grupo de pacientes renais crônicos com diagnóstico confirmado de depressão, visto que o diagnóstico atual de depressão foi relatado pelo paciente, carecendo de maiores investigações de modo a confirmar ou não essas hipóteses. Sugere-se para pesquisas futuras a aplicação da EBADEP-HOSP-AMB em amostras de pacientes com diagnóstico de outras doenças crônicas, além da aplicação da escala com outros instrumentos de avaliação da sintomatologia depressiva. 


\section{DECLARAÇÃO DE CONFLITOS DE INTERESSE}

Não há conflito de interesses.

\section{REFERÊNCIAS}

American Educational Research Association, American Psychological Association, National Council on Measurement in Education (2014). Standards for Educational and Psychological Testing. Washington, DC: American Psychologichal Association.

American Psychiatric Association. (2002). Manual Diagnóstico e Estatístico de Transtornos Mentais DSM-IV-TR. (4a ed.). Porto Alegre, RS: Artmed.

American Psychiatric Association. (2014). Manual Diagnóstico e Estatístico de Transtornos Mentais DSM-5. (5a ed.). Porto Alegre: Artmed.

Anderson, E. R., \& Hope, D. A. (2008). A review of the tripartite model for understanding the link between anxiety and depression in youth. Clinical Psychology Review, 28(2), 275-287. doi:10.1016/j.cpr.2007.05.004

Baptista, M. N. (2012). Escala Baptista de Depressão versão Adulto - EBADEP-A. São Paulo, SP: Vetor.

Baptista, M. N. (2013). Escala Baptista de Depressão - (Versão Ambulatório) EBADEP-HOSP-AMB. Relatório Técnico não publicado. Programa de PósGraduação Stricto-Sensu em Psicologia da Universidade São Francisco. Itatiba. São Paulo.

Bastos, M. G., Bregman, R., \& Kirsztajn, G., M. (2010). Doença renal crônica: Frequente e grave, mas também prevenível e tratável. Revista da Associação Médica Brasileira, 56(2), 248-253. doi:10.1590/S010442302010000200028

Bautovich, A., Katz, I., Smith, M., Loo, C. K., \& Harvey, S. B. (2014). Depression and chronic kidney disease: A review for clinicians. Australian \& New Zealand Journal of Psychiatry, 48(6), 530-541. doi: $10.1177 / 0004867414528589$

Beck, A. T., Rush, A. J, Shaw, F. B., \& Emery, E. (1997). Terapia cognitiva da depressão. Porto Alegre, RS: Artes Médicas.

Botega, N. J., Bio, M. R., Zomignani, M. A., Garcia Júnior, C., \& Pereira, W. A. (1995). Transtornos do humor em enfermaria de clínica média e validação de escala de medida (HAD) de ansiedade e depressão. Revista de Saúde Pública, 29(5), 355-363. doi: 10.1590/S0034-89101995000500004

Carneiro, A. M., \& Baptista, M. N. (2016). Escala de Pensamentos Depressivos (EPD). São Paulo, SP: Hogrefe.

Castillo, A. R. G., Recondo, R. Asbahr, F. R., \& Manfro, G. G. (2000). Transtornos de ansiedade. Revista Brasileira de Psiquiatria, 22(2), 20-23. doi: $10.1590 /$ S1516-44462000000600006 
Clark, L. A., \& Watson, D. (1991). Tripartite model of anxiety and depression: Psychometric eveidence and taxonomic implications. Journal of Abnormal Psychology, 100(3), 316-336. doi:10.1037/0021-843X.100.3.316

Cohen, J. (1988). Statistical Power Analysis for the Behavioral Sciences. New York, NY: Academic Press.

Cremasco, G. S., \& Baptista, M. N. (2017). Análise de estrutura interna da Escala Baptista de Depressão (Versão Hospital-Ambulatório) EBADEP-HOSP-AMB. Manuscrito não publicado.

Dancey, C. P. \& Reidy, J. (2006). Estatística sem matemática para psicologia usando SPSS para Windows. Porto Alegre, RS: Artmed.

Dias, D. R., Shiozawa, P., Miorim, L. A., \& Cordeiro, Q. (2015). Prevalência de sintomas depressivos e ansiosos em pacientes com doença renal crônica em programa de hemodiálise: Um estudo transversal. Arquivos Médicos dos Hospitais e da Faculdade de Ciências Médicas da Santa Casa São Paulo, $60(2), 65-71$.

Feroze, U., Martin, D., Kalantar-Zadeh, K., Kim, J. C., Reina-Patton, A., \& Kopple, J. D. (2012). Anxiety and depression in maintenance dialysis patients: preliminary data of a cross-sectional study and brief literature review. Journal of Renal Nutrition, 22(1), 207-210. doi: 10.1053/j.jrn.2011.10.009

Ferster, C. B., Culbertson, S., \& Boren, M. C. (1977). Depressão clínica. In C. B. Ferster, S. Culbertson, \& M. C. Boren, Princípios do Comportamento (pp. 699-725). São Paulo, SP: Hucitec.

First, M. B., Spitzer, R. L., Gibbon, M., \& Williams, J. B. W. (1996). Structured clinical interview for DSM-IV axis I disorders, clinician version (SCID-CV). Washington, D.C: American Psychiatric Press.

Lynch, J., Moore, M., Moss-Moris, R., \& Kendrick, T. (2011). Are patient beliefs important in determining adherence to treatment and outcome for depression? Development of the beliefs about depression questionnaire. Journal of Affective Disorders, 133(1), 29-41. doi:10.1016/j.jad.2011.03.019

Marques, M. A. B. (2016). Sintomas da depressão após lesão encefálica: Evidências de validade para EBADEP-HOSP-AMB. (Tese de Doutorado). Universidade São Francisco, Itatiba, SP.

Messias, C. R. (2014). Sintomatologia Depressiva e associações com dor crônica, saúde geral e suporte social em pacientes fibromiálgicos e oncológicos. (Dissertação de mestrado). Universidade São Francisco, Itatiba, SP.

Organização Mundial da Saúde (OMS). (1993). Classificação dos transtornos mentais e do comportamento - CID-10: Descrições e diretrizes diagnósticas. (10a ed.). Porto Alegre, RS: Artes Médicas. 
Pinto, J. C., Martins, P., Pinheiro, T. B., \& Oliveira, A. C. (2015). Ansiedade, depressão e stresse: Um estudo com jovens adultos e adultos portugueses. Psicologia, Saúde \& Doenças, 16(2), 148-163. doi.org/10.15309/15psd160202

Prieto, G., \& Muñiz, J. (2000). Un modelo para evaluar la calidad de los test utilizados em España. Papeles del Psicólogo, 77, 65-72.

Stasiak, C. E. S., Bazan, K. S., Kuss, R. S., Schuinski, A. F. M., \& Baroni, G. (2014). Prevalência de ansiedade e depressão e suas comorbidades em pacientes com doença renal crônica em hemodiálise e diálise peritoneal. Jornal Brasileiro de Nefrologia, 36(3), 325-331. doi:10.5935/01012800.20140047

Theofilou, P. (2011). Depression and anxiety in patients with chronic renal failure: The effect of sociodemographic characteristics. International Journal of Nephrology, 2011, 1-6. doi:10.4061/2011/514070

Watson, D. (2009). Differentiating the mood and anxiety disorders: A quadripartite model. Annual Review of Clinical Psychology, 5(1), 221-247. doi: 10.1146/annurev.clinpsy.032408.153510

Watson, D., Weber, K., Assenheimer, J. S., Clark, L. A., Strauss, M. E., \& McCormick, R. A. (1995). Testing a tripartite model: I. Evaluating the convergent and discriminant validity of anxiety and depression symptom scales. Journal of Abnormal Psychology, 104(1), 3-14. doi:10.1037/0021843X.104.1.3

World Health Organization (WHO) (2017). Depression and other common mental disorders: Global health estimates. Recuperado de http://www.who.int/mental_health/management/depression/prevalence_glo bal_health_estimates/en/

Zigmond, A. S., \& Snaith, R. P. (1983). The Hospital Anxiety and Depression Scale. Acta Psychiatrica Scandinavica, 67(6), 361-370. doi:10.1111/j.16000447.1983.tb09716.x 
Sobre os autores

Gabriela da Silva Cremasco é psicóloga. Mestre e Doutoranda pelo Programa de Pós-Graduação Stricto Sensu em Psicologia com ênfase em Avaliação Psicológica pela Universidade São Francisco como Bolsista pela Coordenação de Aperfeiçoamento de Pessoal de Nível Superior (CAPES). E-mail: gabisilva10@hotmail.com

Makilim Nunes Baptista é psicólogo. Doutor em Psiquiatria e Psicologia Médica pela Universidade Federal de São Paulo. Professor do Programa de PósGraduação Stricto Sensu em Psicologia da Universidade São Francisco e Bolsista Produtividade pelo Conselho Nacional de Desenvolvimento Científico e Tecnológico. E-mail: makilim01@gmail.com

A contribuição de cada autor pode ser atribuída como se segue: G.C. e M.N. contribuíram para a conceitualização, investigação e visualização do artigo; G.C. e M.N. foram responsáveis pela obtenção de financiamento; G.C. fez a redação inicial do artigo (rascunho) e G.C. e M.N. são os responsáveis pela redação final (revisão e edição).

Os autores agradecem a CAPES pelo financiamento da pesquisa.

Recebido em: 10/12/2017

$1^{\text {a }}$ revisão em: $10 / 04 / 2018$

Aceito em: $17 / 04 / 2018$ 